\title{
The Predictive Value of Selenium in Diagnosis of Gestational Diabetes: A Nested Case-Control Study
}

This article was published in the following Dove Press journal:

International Journal of General Medicine

\author{
Zeinab Moshfeghy' \\ Khadigeh Bashiri ${ }^{2}$ \\ Mohammad HDabbaghmanesh ${ }^{3}$ \\ Marzieh Akbarzadeh ${ }^{4}$ \\ Nasrin Asadi ${ }^{5}$ \\ Mehrab Sayadi ${ }^{6}$ \\ 'Community Based Psychiatric Care \\ Research Center, Department of \\ Midwifery, School of Nursing and \\ Midwifery, Shiraz University of Medical \\ Sciences, Shiraz, Iran; ${ }^{2}$ Department of \\ Midwifery, School of Nursing and \\ Midwifery, Shiraz University of Medical \\ Sciences, Shiraz, Iran; ${ }^{3}$ Department of \\ Internal Medicine, Endocrine and \\ Metabolism Research Center, Shiraz \\ University of Medical Sciences, Shiraz, \\ Iran; ${ }^{4}$ Maternal-Fetal Medicine Research \\ Center, Department of Midwifery, School \\ of Nursing and Midwifery, Shiraz \\ University of Medical Sciences, Shiraz, \\ Iran; ${ }^{5}$ Department of Obstetrics and \\ Gynecology, School of Medicine, Shiraz \\ University of Medical Sciences, Shiraz, \\ Iran; ${ }^{6}$ Department of Bio-Statistics, Shiraz \\ University of Medical Sciences, Shiraz, \\ Iran
}

Correspondence: Marzieh Akbarzadeh Maternal-Fetal Medicine Research

Center, Department of Midwifery, School of Nursing and Midwifery, Shiraz University of Medical Sciences, Shiraz, Iran

Tel $+987 \mathrm{II}-6474250$

Fax $+98711-647425$

Email marzieh.akhbar@gmail.com
Aim: The present study was conducted to determine the predictive value of Selenium (Se) in the diagnosis of Geatational diabetes Mellitus (GDM).

Methods: This is a nested case-control study with 636 normal pregnant mothers in their 11th-13th weeks. Gestational diabetes screening was done in weeks 24-28. Twenty-five individuals were detected as GDM, and for every GDM two gestational age-matched normal pregnant women were selected. The blood selenium level was measured in both groups.

Results: The serum Se level in the case group was lower than that of the control group $(50.60 \pm$ 10.88 versus $66.02 \pm 10.57$ ) in the first trimester. Also, in the second trimester, Se was lower in the case group $(39.87 \pm 10.23$ versus $63.17 \pm 10.22)$. The best cut-off point for selenium in order to predict the incidence of gestational diabetes in our study was 48.2. Pregnant women with selenium levels below 48.2 were more likely to develop gestational diabetes.

Conclusion: Serum selenium was lower in the GDM subjects compared with age-matched control group; the clinical concept and mechanism of this finding need to be investigated through further studies.

Keywords: diabetes mellitus, gestational, prenatal, pregnancy, selenium

\section{Introduction}

Gestational Diabetes mellitus (GDM) is referred to as having various degrees of glucose intolerance with onset or recognition during pregnancy. ${ }^{1-4} 1-14 \%$ of all pregnancies are accompanied with GDM, inducing short- and long-term maternal and prenatal complications. ${ }^{1,5}$ During natural pregnancy, Insulin resistance increases parallel with the rise in oxidative stress. It, eventually, results in antioxidant level reduction. $^{6,7}$

The aforesaid conditions are more observed in the GDM affected women. Glucose oxidation, protein glycation, as well as lipid peroxidation in women suffering from GDM cause free radical accumulation. ${ }^{6,8}$ It is argued that there is a positive correlation between erythrocyte glutathione peroxidase activities and Insulin resistance. This correlation reveals that oxidative stress decreases the insulin secretion and increases the insulin resistance. Accordingly, this correlation is connected to Type 2 diabetes. 6,7

Selenium (Se), an essential trace element, is considered a key component of numerous selenoproteins in an antioxidant defense system. Glutathione peroxides consist of a group of enzymes supporting the body against oxidative stress. Glutathione peroxides have selenium in the form of selenocysteine in the catalytic site. ${ }^{9}$ It is also assumed that selenium with properties like insulin can have antidiabetic function. ${ }^{6}$ 
Recently, the result of a review article and meta-analysis carried out by Asgari et al revealed that Se concentration of women with gestational diabetes is substantially lower than that of the normal pregnant women. ${ }^{6}$ Kilinc et al's research showed that serum selenium level in women with gestational diabetes and those with glucose intolerance is lower than that of the normal pregnant women. ${ }^{10}$ Tan et al's survey also indicated that Se level in gestational diabetes and glucose intolerance was lower than that of the normal pregnant women. ${ }^{11}$ Han reached the conclusion that there was a link between low Se level and gestational diabetes. Asemi in a clinical trial indicated that Se supplementation among pregnant women with gestational diabetes resulted in improved glucose homeostasis as well as reduced inflammation and improved oxidative stress. ${ }^{12}$

The above-mentioned surveys showed that low level of selenium in women is related to gestational diabetes. It should be noted that none of these studies has looked into the casual relationship of gestational diabetes and selenium. This study is an attempt to evaluate the predictive value of selenium in gestational diabetes. Such research has not been conducted, as far as the authors know, in the last 20 years.

\section{Method}

\section{Setting}

This study is a nested case-control study. First, 636 gestational age cohorts were enrolled in the study and at the first pregnancy visit, the required samples were obtained. Then, the study sample was followed up until the outcome, which is gestational diabetes. After the outcome, the case group and those who were negative for gestational diabetes were included in the main phase of the study. The case is called a nested witness. This study was performed to compare the plasma levels of selenium in healthy primiparous women with gestational diabetes who referred to selected clinics Hafez educational and therapeutic center and Comprehensive Medical Genetic Center (CMGC) affiliated to Shiraz University of Medical Sciences in 2013-2014.

\section{Study Population}

On the basis of the former results, the sample size of gestational diabetes and normal groups was 25 and 50 subjects, respectively. The number of the subjects was calculated using the following formula $n^{\prime}=\frac{\left(z_{1-\frac{\alpha}{2}+z_{1-\beta}}\right)^{2}}{\left(\frac{d}{2 \delta}\right)^{2}}(\delta=/ 093$,

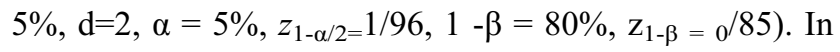

a prospective study design, the required sample size in the case group with a confidence level of $95 \%, p=5 \%$ (incidence rate of Diabetes), for the purpose of $n$ numbers using the formula, $n=\left[A+\sqrt{A^{2}+\frac{n^{\prime}}{p}}\right]^{2} ; 636$ was obtained. In this formula, $n=\frac{\left|Z_{\alpha}\right|}{2} \sqrt{\frac{1-P}{P}}$

Therefore, 636 eligible primigravida women were included in this study from March 2014 to January 2015, with the following inclusion (placement) criteria: singleton pregnancy, age lower than 30 years old (because age is associated with the incidence of diabetes, we have to control the role of the age. In the onset of gestational diabetes, women under the age of 30 were studied., gestational age less than 14 weeks based on the first trimester ultrasound exam, none-smokers, no history of recognized internal diseases such as hypertension, diabetes, kidney disease, cardiovascular disease, etc., no personal history of glucose intolerance, no personal and family history of diabetes, neither personal nor family history of deep venous thrombosis, with $\mathrm{BMI}<30\left(\mathrm{~kg} / \mathrm{m}^{2}\right)$, no history of chronic disease or vitamin deficiency, lack of taking any medication (except for iron supplementation) or any types of multivitamins containing selenium during the previous year, and living in Shiraz during the previous 2 years. All participants had gestational age under 14 weeks at the beginning of the study and were followed until 24-28 weeks of pregnancy. The study group was requested to fill out a questionnaire to get their demographic data and their physical activities before pregnancy. Exclusion criteria included use of multivitamin supplementation containing Se during the study; lack of correlation between gestational age based on the last menstrual period and sonographic exam of first trimester; no chronic disease such as kidney, thyroid, hypertension and cardiovascular diseases; preterm labor or abortion or any complication prior to 28 gestational weeks; no presentation to prenatal care unit; and withdrawal from the study. The gestational age of the subjects was lower than 14 weeks. Their cases were followed up till 24-28 gestational week. All research units were asked to fill out the questionnaires to obtain their demographic information as well as physical activities prior to gestation.

\section{Matching Process Between the Case and Control Subjects}

Sampling was done by using a convenience sampling method; then, out of 636 patients who were included in 
the study, about 25 subjects were diagnosed as gestational diabetes mellitus, which was considered as the case group. For the control group, we selected the gestational age of the case and healthy mothers. Then, for each case based on gestational age, we arranged the healthy groups and randomly selected one of them to match the gestational age according to the selected control group.

\section{Variable Considerations}

Anthropometric parameters including weight, height, and body mass index were measured. Weight was measured using a standard scale while the subjects were in light clothing. Body mass index was calculated as weight (in kilograms) divided by the squared height (in meters). The height was measured by using tape measure.

\section{Outcome}

\section{Blood Sample Collection and Analysis}

For the first phase of the study, 5-mL venous blood was taken from each subject in the first trimester. The serum was separated within $1 \mathrm{hr}$ after blood sampling by centrifugation. Then, all the samples were coded and transferred to Endocrine and Metabolism Research Center of Shiraz University of Medical Sciences. The serum was frozen at $-70^{\circ} \mathrm{C}$ and remained intact in the site until the end of the second phase of sampling. All the subjects between 24 and 28 weeks of pregnancy received $50 \mathrm{~g}$ oral glucose challenge test. If glucose was $\geq 140 \mathrm{mg} / \mathrm{dL}$, the participants underwent a $100 \mathrm{~g}$ OGTT. GDM was diagnosed if two glucose values were above the thresholds defined by Carpenter and Coustan (fasting plasma glucose $>95 \mathrm{mg} / \mathrm{dL}$, 1-h post load glucose (PLG) $>180 \mathrm{mg} / \mathrm{dL}, 2-\mathrm{h}$ PLG $>155 \mathrm{mg} / \mathrm{dL}$ and $3-\mathrm{h}$ PLG $>140 \mathrm{mg} / \mathrm{dL}$ ). 25 women were diagnosed with GDM and for each GDM patient 2 matched gestational age pregnant women were selected from healthy pregnant women. Then, the second stage of blood samples from gestational diabetes and selected healthy individuals were taken.

\section{Serum Selenium Levels}

The third phase of survey was launched by measuring the selenium of the first and second phases of the case and control groups. The experiments were performed by using a GBC atomic absorption spectrometer (AAS 932 - HG3000-AUS) equipped by hydride generation module. The operated parameters for the metal of interest were set as recommended by the manufacturer. Selenium was determined by hydride generation atomic absorption spectrometry (HG-AAS) and selenium (Si) hollow cathode lamp operating at the current of $4 \mathrm{~mA}$, the wavelength of $196 \mathrm{~nm}$, and the spectral bandwidth of $2 \mathrm{~nm}$.

\section{Statistical Analysis}

In the present study, statistical analysis was performed using SPSS, version 20. Values were expressed as mean \pm standard deviation. An independent-samples $t$-test was used to compare the quantitative variables including age, height, weight, BMI, gestational age at the beginning of the study, mean of the glucose challenges and selenium level. Chisquare test was applied for the qualitative variables including the jobs, education and physical activities between the patient and control groups. Kolmogorov Smirnov Test was used for data normality assessment. Pearson Correlation Test was applied for evaluation of the correlation of variables (the mean of GCT and BMI). Logistic Regression Analysis was conducted to find the relationship between the selenium level and gestational diabetes with BMI control variables. ROC curve was used to determine the best cut-off point. A p-value $<0.05$ was considered significant.

\section{Result}

Six hundred and thirty-six women were included in this study. Twenty-five pregnant women underwent abortion and 6 people had preterm labor. No significant difference was detected between the two groups concerning the mean age, education level, pre-pregnancy physical activity and gestational age, whereas there was a significant difference between the groups in job ( $p$-value $<0.046$ )

Pre-pregnancy mean weight was more in the GDM group in comparison with the normal group and a statistically significant difference was observed between the two groups regarding BMI (Table 1).

The mean $1 \mathrm{hr}$ glucose level (with $50 \mathrm{~g}$ glucose) in the 28-24 weeks of gestation in the case and control groups was statistically significant $(\mathrm{p}=0.0 .001)$ (Table 2$)$. Table 3 shows the mean fasting blood glucose and glucose tolerance of $3 \mathrm{hrs}$ ( $1 \mathrm{hr}, 2 \mathrm{hrs}$ and $3 \mathrm{hrs}$ ) in the case group (Table 3 ).

Serum Se concentration in the case group was significantly lower than that of the normal group during both first $(50.60 \pm 10.88$ versus $66.02 \pm 10.57)$ and second trimesters $(39.87 \pm 10.23$ versus $63.17 \pm 10.22)$. Moreover, Se level in the second trimester was significantly lower than that of the first trimester in the case and control groups. Se level decreased in the second trimester in the case and control groups $(10.68 \pm 3.61,2.84 \pm 1.88)$, respectively, in comparison to the first trimester (Tables 4 and 5). Besides, pre-pregnancy 
Table I Demographic Characteristics of the Patients and Control Groups

\begin{tabular}{|c|c|c|c|c|c|}
\hline \multirow[t]{2}{*}{ Variables } & \multicolumn{2}{|c|}{$\begin{array}{l}\text { GDM } \\
(n=25)\end{array}$} & \multicolumn{2}{|c|}{$\begin{array}{l}\text { Normal } \\
(n=50)\end{array}$} & \multirow[t]{2}{*}{$P$ value } \\
\hline & Mean & SD & Mean & SD & \\
\hline Age & 25.76 & 3.655 & 25.66 & 3.526 & 0.909 \\
\hline Gestational Age & 12.4 & 0.61 & 12.16 & 0.65 & 0.445 \\
\hline At entry & & & & & \\
\hline Length & 161.52 & 7.04 & 162.3 & 5.28 & 0.592 \\
\hline Weight & 66.43 & 14.45 & 61.02 & 8.18 & $0.042 *$ \\
\hline \multirow[t]{2}{*}{ BMI } & 25.36 & 5.31 & 22.77 & 2.85 & $0.029 *$ \\
\hline & $\mathbf{N}$ & $\%$ & $\mathbf{N}$ & $\%$ & \\
\hline Job & & & & & $0.46 *$ \\
\hline Householder & 25 & 100 & 42 & 84 & \\
\hline Employed & 0 & & 8 & 16 & \\
\hline Education & & & & & $0.44 *$ \\
\hline Diploma and under & 12 & 48 & 22 & 44 & \\
\hline University & 13 & 52 & 28 & 56 & \\
\hline Physical activity & & & & & 0.259 \\
\hline Home work & 14 & 56 & 31 & 62 & \\
\hline Walking & 7 & 28 & 5 & 10 & \\
\hline Light exercise & 4 & 16 & 9 & 18 & \\
\hline Professional sport & 0 & 0 & 2 & 4 & \\
\hline $\begin{array}{l}\text { Homework and } \\
\text { walking }\end{array}$ & 0 & 0 & 3 & 6 & \\
\hline
\end{tabular}

Note: *P values are significant.

Table 2 Mean Blood Glucose I hr (50 g Glucose) in 24-28 Weeks in Both Groups

\begin{tabular}{|l|l|l|l|l|l|}
\hline \multirow{2}{*}{ Variables } & \multicolumn{2}{|l|}{ GDM** $(\mathbf{n}=\mathbf{2 5})$} & \multicolumn{2}{|l|}{ Normal $(\mathbf{n}=\mathbf{5 0})$} & \multirow{2}{*}{ P value } \\
\cline { 2 - 5 } & Mean & SD & Mean & SD & \\
\hline GCT $^{*}$ & 163.48 & 14.84 & 105.30 & 14.49 & $0.001<$ \\
\hline
\end{tabular}

Notes: *Glucose challenge test; **Gestational diabetes mellitus.

Table 3 Mean of Fasting Blood Glucose and 3 hrs' Glucose Tolerance (I hr, 2 hrs and $3 \mathrm{hrs}$ ) in the Case Group

\begin{tabular}{|l|l|l|}
\hline \multirow{2}{*}{ Variables } & GDM & SD \\
\cline { 2 - 3 } & Mean & 24.54 \\
\hline FBS & 95.40 & 17.55 \\
GTT I hr & 201.48 & 24.5 \\
GTT 2 hrs & 163.44 & 22.12 \\
GTT 3 hrs & 125.52 & \\
\hline
\end{tabular}

weight in the case group was more than the control group and body mass index was higher in the case group than in the control group (Table 1). The relationship between body mass index and GCT was calculated using Pearson correlation coefficient that was not statistically significant (Table 6).

By using ROC curve, the best cut-off point for prediction of gestational diabetes was obtained 48.2. Sensitivity and specificity of this point were $0.83,0.94$, respectively, being statistically significant. The area under the curve was 0.93 which was statistically significant (Figure 1).

Pearson correlation showed that BMI and GCT means were not statistically significant between the two groups. Logistic Regression Analysis was used to evaluate the effect of Se and BMI on the outbreak of gestational diabetes. According to the result of this analysis, the effect of BMI on the outbreak of diabetes was not statistically significant.

\section{Discussion}

According to our results, serum Se level decreased during pregnancy, and its reduction was significant in the gestational diabetes women. Se level in the first trimester of pregnancy was lower in those individuals who later had gestational diabetes than the normal group. Se level in diabetic women was lower than that of the normal group in the 24-28 weeks.

Selenium is an antioxidant material helping to prevent the adverse effects of free radicals. Glutathione peroxidase (GP) which holds about $60 \%$ of the selenium in the body is an antioxidant enzyme helping to detoxify the body and protecting the cell against oxidative impairment. ${ }^{10}$

Evidence shows that serum selenium level reduces during pregnancy. ${ }^{6,13,14}$ The reasons for reduction may be hemodilutional phenomenon in pregnancy, the rise of fetal requirements, and deposition in the placenta. ${ }^{6,10,11,15}$ Furthermore, owing to the rise of lipid peroxidation during pregnancy, the activity of antioxidants such as glutathione and erythrocyte glutathione peroxidase increases; therefore, the level of serum Se decreases, especially in the third trimester. ${ }^{6,7}$ In women affected with GDM, the overproduction of free radicals induces the impairment of radical scavenger function mechanisms. ${ }^{16} \mathrm{~A}$ lower level of selenium has been explained in GDM, and it has been indicated that there was an inverse relationship with this trace element status and glucose tolerance..$^{10,11,17-19}$

In numerous researches, Se level was measured during the 24-28 weeks. Kilinc et al showed that Se level in women with gestational diabetes and glucose intolerance is lower than that of the control group. The mean level of Se in gestational diabetes, glucose intolerance and control group was $34.7 \pm 8.7,39.9 \pm 6.5$, and $50.7 \pm 6.5$, respectively. ${ }^{10}$ Heilan 
Table 4 Bio-Variable and Multivariable Logistic Regression on Selenium and Other Covariates

\begin{tabular}{|l|l|l|l|l|l|l|}
\hline \multirow{2}{*}{ Variables } & \multicolumn{2}{l|}{ Bio Variable (Unadjusted) } & \multicolumn{3}{l|}{ (Adjusted) Multivariable } \\
\cline { 2 - 7 } & Coefficient & P value & OR (95\% Cl) & Coefficient & P value & OR (95\% CI) \\
\hline Age & -0.008 & 0.908 & $0.992(0.865-1.137$ & 0.059 & 0.568 & $1.061(0.866-0.299)$ \\
BMI & -0.172 & $\mathbf{0 . 0 1 6}$ & $0.842(0.732-0.968$ & -0.150 & 0.115 & $0.860(0.714-1.037)$ \\
Selenium & 0.146 & $<\mathbf{0 . 0 0 I}$ & $1.157(1.0179-1.242$ & 0.140 & $<0.001$ & $1.151(1.07 I-1.236)$ \\
\hline
\end{tabular}

Note: The bold values represent the significant difference in $\mathrm{p}$ values.

Table 5 Changes of Serum Selenium Level in Gestational Diabetes and Normal Pregnant Women in the First and Second Trimester

\begin{tabular}{|l|l|l|l|}
\hline Selenium & GDM $(\mathbf{n}=\mathbf{2 5})$ & Normal $(\mathbf{n}=\mathbf{5 0})$ & P value \\
\hline First trimester & $50.60 \pm 10.88$ & $66.02 \pm 10.57$ & $<0.001$ \\
$24-28$ week & $39.87 \pm 10.23$ & $63.17 \pm 10.22$ & $<0.001$ \\
Mean change & $-10.68 \pm 3.61$ & $-2.84 \pm 1.88$ & \\
P value & $<0.001$ & $<0.001$ & \\
\hline
\end{tabular}

Table 6 Correlation Between GCT and BMI in the Case and Control Groups

\begin{tabular}{|l|l|l|}
\hline Group & Case & Control \\
\hline Pearson correlation & -0.285 & 0.072 \\
P-value & 0.167 & 0.619 \\
\hline
\end{tabular}

et al reached the conclusion that Glutathione peroxides activity and level of Se may contribute to the pathogenesis of gestational diabetes. The Se levels in gestational diabetes and control group were $0.0620 \pm 0.0224$, and $0.0783 \pm 0.0209$, respectively. Furthermore, Glutathione peroxides activity in the case group was higher than that of the control group. ${ }^{20}$ Other studies revealed that Se level in gestational diabetic women was lower than that of the control group. ${ }^{11,20,21}$ The result of our research confirms other researcher's achievements. The result of Hawkes' study revealed that the Se level diminished at 12-34 weeks of gestation. However, this reduction was not statistically significant, whereas in our research, the Se level in both case and control groups decreased and this reduction was significant in the control group. In Hawke's research, 22 pregnant women were included in 12th week, one of whom was suffering from glucose intolerance and the rest of the subjects were healthy. Five subjects were found to have glucose intolerance and 1 had gestational diabetes. The sample size in the Hawke's study was lower than that of the present study; furthermore, every subject was considered as her own control. ${ }^{17}$ However, in our study, 636 eligible primigravida women were included. All participants had gestational age under

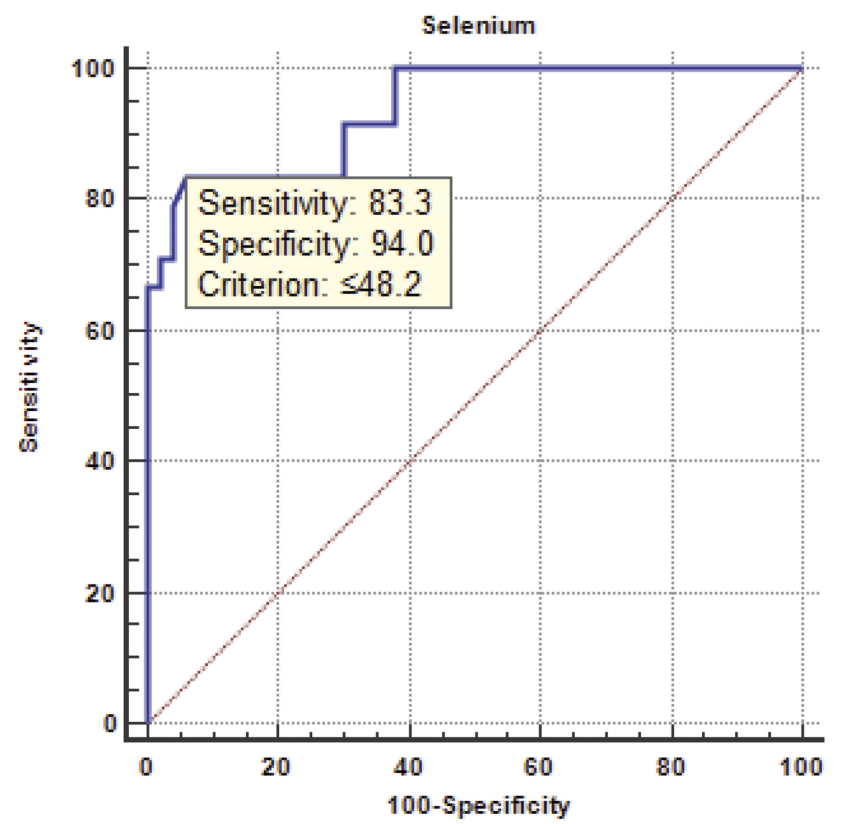

Figure I ROC curve showed that gestational diabetic could be predicted by the selenium level with high accuracy. A value is equal to or lower than 48.2 as diabetic patient. The area under the curve was 0.93 and $95 \%$ confidence interval was 0.85 to 0.95 that was statistically significant. Sensitivity and specificity of this point were 83.3and $94.0 \%$, respectively. Also, positive predictive value (Ppv) and negative predictive value (NPV) were $86.9 \%$ and $92.1 \%$.

14 weeks at the beginnings of the study and were followed until 24-28 weeks of pregnancy.

Twenty-five people were known as GDM and 50 normal pregnant women were the control group. Moreover, mean Se level $(111 \pm 12,126 \pm 15)$ in 12,34 weeks was higher than our mean; this can be due to the difference of selenium in the soil in different areas. Askari et al found a negative correlation between selenium and gestational diabetes, especially in the third trimester. ${ }^{6}$ They could not detect a causal relationship between Se and GDM due to the design of the research (casecontrol or cross-sectional). On the basis of this meta-analysis, it is unknown that decrease in the serum $\mathrm{Se}$ is a predictive value of gestational diabetes and glucose intolerance or pregnant women with hyperglycemia have low Se level.

Also, in further studies, the results showed that some of them had a lower serum level of selenium, which is in the 
same line with our study. ${ }^{22-24}$ However, some studies have shown that the level of diabetes in diabetic patients is greater than the control group, which is in contrast to our study. ${ }^{25-27}$ And in some studies, the serum levels of selenium in diabetic patients and control group did not differ. ${ }^{28}$ And these differences may be due to racial differences and other interfering factors that require more studies. Selenium content also varies in different geographic regions, which is effective in altering the selenium levels. Besides, in the study of Liu X and colleagues, 14 Serum Trace Elements including selenium in pregnancy were studied in Chinese women as to their health and nutrition. The results showed that serum Fe, Zn, and Se concentrations were significantly reduced, but this study has not examined the association between this decline and the incidence of diabetes. ${ }^{29}$

The cut-off points for gestational diabetes were obtained $48.2 \mu \mathrm{g} / \mathrm{L}$ with a sensitivity of $83.3 \%$ and specificity of $94 \%$. In previous studies, only the Se value was compared in the case and control groups and the cutoff point was not reported. Two studies were done in Iran, reporting the cut-off points for preeclampsia. Ghaemi in a research conducted in Shiraz in 2013 reported that $62.2 \mu \mathrm{g} / \mathrm{L}$ has been determined as the Se cut-off point. ${ }^{30}$ Maleki et al obtained $60 \mu \mathrm{g} / \mathrm{L}$ as the cutoff point. ${ }^{31}$ The difference of our cut-off point from the mentioned studies can indicate that Se level decreases significantly in GDM, in comparison with preeclampsia.

\section{Implications for Practice}

If further studies on selenium and gestational diabetes are carried out and the findings of this study are confirmed, the incidence of gestational diabetes can be reduced by administration of selenium supplement to pregnant women. In addition, selenium supplements can be used to treat pregnant women with gestational diabetes.

The strength of this study is that, unlike other crosssectional or case-control studies, we designed a Nested Case-Control study of serum selenium level. Furthermore, the present research made a comparison between the Se level of the first trimester and 24-28 weeks of pregnancy. This comparison was the distinctive feature of the present survey with previous surveys. The change process of selenium levels was also examined and we could determine the best cut-off point for developing gestational diabetes.

The limitation of this study is that the incidence of diabetes in the study population was low. It could also be due to the fact that the women participating in the study had an average risk of gestational diabetes. Another limitation of our study was the measurement of selenium in patients with glucose intolerance that was due to the low cost.

\section{Conclusion}

Due to the low serum selenium levels in the case group in the first trimester compared to the control group and more significant reduction of selenium in women with GDM during pregnancy, it can be concluded that the serum selenium was lower in GDM subjects compared with agematched control group; also, the clinical concept and mechanism of this finding need to be investigated through further studies. The reduced serum levels may be effective in prediction of GDM. Due to the cut-off point obtained in this study, it can be said that pregnant women who have serum selenium levels less than $48 / 2 \mu \mathrm{g} / \mathrm{L}$ in the first trimester are more likely to be diagnosed with gestational diabetes. By applying Se to the routine tests at the first prenatal care, we can identify the individuals with low levels of selenium; moreover, by prescribing selenium supplements we can prevent these women from gestational diabetes. Further studies with larger sample sizes are needed to examine the role of selenium in the onset of gestational diabetes and support our findings.

\section{Ethical Approval}

The present research was approved by the ethics committee of Shiraz University of Medical Sciences and written informed consents were obtained from all participants (proposal No: 6905 ethical code: IR. sums. REC S6905). This study was conducted in accordance with the Declaration of Helsinki.

\section{Acknowledgments}

This article is a part of Khadijeh Bashiri's thesis under the code number of 6905 . The authors would like to thank the vice-chancellor for research of Shiraz University of Medical Sciences for financial support. They also extend my sincere appreciation to the president of the CMGC, Dr Tabei and the personnel of the center for their contributions in sampling phase. The authors would like to thank Development of Clinical Research Center of Nemazee Hospital and Dr. Nasrin Shokrpour for editorial assistance.

\section{Funding}

The study was financially supported by the Research Vicechancellor of Shiraz University of Medical Sciences, Shiraz, Iran. Guaranteeing author's name: MA. 


\section{Disclosure}

The authors report no conflicts of interest in this work.

\section{References}

1. Yeral MI, Ozgu-Erdinc AS, Uygur D, Seckin KD, Karsli MF, Danisman AN. Prediction of gestational diabetes mellitus in the first trimester, comparison of fasting plasma glucose, two-step and one-step methods: a prospective randomized controlled trial Endocrine. 2014;46(3):512-518. doi:10.1007/s12020-013-0111-z

2. American Diabetes Association. Standards of medical care in diabetes-2011. Diabetes Care. 2011;34 Suppl 1:S11-S61. doi:10.2337/ dc11-S011

3. Nolan CJ. Controversies in gestational diabetes. Best Pract Res Clin Obstet Gynaecol. 2011;25(1):37-49. doi:10.1016/j.bpobgyn.2010. 10.004

4. Farrar D, Duley L, Lawlor DA. Different strategies for diagnosing gestational diabetes to improve maternal and infant health. Cochrane Database Syst Rev. 2011;10:CD007122.

5. American Diabetes Association. Diagnosis and classification of diabetes mellitus. Diabetes Care. 2011;34 Suppl 1:S62-S69. doi:10.23 37/dc11-S062

6. Askari G, Iraj B, Salehi-Abargouei A, Fallah AA, Jafari T. The association between serum selenium and gestational diabetes mellitus: a systematic review and meta-analysis. J Trace Elem Med Biol. 2015;29:195-201. doi:10.1016/j.jtemb.2014.09.006

7. Chen X, Scholl TO, Leskiw MJ, Donaldson MR, Stein TP. Association of glutathione peroxidase activity with insulin resistance and dietary fat intake during normal pregnancy. $J$ Clin Endocrinol Metab. 2003;88(12):5963-5968. doi:10.1210/jc.2003-030544

8. Buchanan TA, Xiang AH. Gestational diabetes mellitus. J Clin Invest. 2005;115(3):485. doi:10.1172/JCI200524531

9. Gao H, Hagg S, Sjogren P, Lambert PC, Ingelsson E, van Dam RM, Serum selenium in relation to measures of glucose metabolism and incidence of Type 2 diabetes in an older Swedish population. Diabet Med. 2014;31(7):787-793. doi:10.1111/dme.2014.31.issue-7

10. Kilinc M, Guven MA, Ezer M, Ertas IE, Coskun A. Evaluation of serum selenium levels in Turkish women with gestational diabetes mellitus, glucose intolerants, and normal controls. Biol Trace Elem Res. 2008;123(1-3):35-40. doi:10.1007/s12011-007-8087-2

11. Tan M, Sheng L, Qian Y, et al. Changes of serum selenium in pregnant women with gestational diabetes mellitus. Biol Trace Elem Res. 2001;83(3):231-237. doi:10.1385/BTER:83:3

12. Asemi Z, Jamilian M, Mesdaghinia E, Esmaillzadeh A. Effects of selenium supplementation on glucose homeostasis, inflammation, and oxidative stress in gestational diabetes: randomized, double-blind, placebo-controlled trial. Nutrition. 2015;31(10):1235-1242. doi:10.1016/j.nut.2015.04.014

13. Kartinih K, Wibowo N. The level of zinc is increasing, while the serum folate level is decreasing, after multi-micronutrient supplementation in pregnant woman. Indones J Obstet Gynecol. 2013;36(4):171-175.

14. Kosanovic M, Jokanovic M, Jevremovic M, Dobric S, Bokonjic D. Maternal and fetal cadmium and selenium status in normotensive and hypertensive pregnancy. Biol Trace Elem Res. 2002;89(2):97-103. doi:10.1385/BTER:89:2

15. Molnar J, Garamvolgyi Z, Herold M, Adanyi N, Somogyi A, Rigo JJ. Serum selenium concentrations correlate significantly with inflammatory biomarker high-sensitive CRP levels in Hungarian gestational diabetic and healthy pregnant women at mid-pregnancy. Biol Trace Elem Res. 2008;121(1):16-22. doi:10.1007/s12011-007-8018-2
16. Lappas M, Hiden U, Desoye G, Froehlich J, Mouzon SH-D, Jawerbaum A. The role of oxidative stress in the pathophysiology of gestational diabetes mellitus. Antioxid Redox Signal. 2011;15 (12):3061-3100.

17. Hawkes WC, Alkan Z, Lang K, King JC. Plasma selenium decrease during pregnancy is associated with glucose intolerance. Biol Trace Elem Res. 2004;100(1):19-29. doi:10.13 85/BTER:100:1

18. Quilliot D, Dousset B, Guerci B, Dubois F, Drouin P, Ziegler O. Evidence that diabetes mellitus favors impaired metabolism of zinc, copper, and selenium in chronic pancreatitis. Pancreas. 2001;22 (3):299-306. doi:10.1097/00006676-200104000-00012

19. Orhan H, Önderoglu L, Yücel A, Sahin G. Circulating biomarkers of oxidative stress in complicated pregnancies. Arch Gynecol Obstet. 2003;267(4):189-195. doi:10.1007/s00404-002-0319-2

20. HaiLan Y, Fang H, XiaoLing S. The relationship among gestational diabetes mellitus, serum selenium level and glutathione peroxidase activity. Maternal Child Health Care China. 2009;24(27): 3788-3789.

21. Tong J, Xing -L-L, Wang H. The Study on the content of selenium of the maternal blood, newborn blood and placental tissue of in women with gestational diabetes mellitus. Chin J Birth Health Heredity. 2006;9:033.

22. Kruse-Jarres JD, Rukgauer M. Trace elements in diabetes mellitus. Peculiarities and clinical validity of determinations in blood cells. J Trace Elem Med Biol. 2000;14(1):21-27. doi:10.1016/S0946-672X (00)80019-X

23. Kumar R. Correlation of Selenium and other antioxidants in diabetic patients with and without complications. Free Radic Antioxid. 2012;2 (1):6-8. doi:10.5530/ax.2012.2.3

24. Ruiz C, Alegria A, Barbera R, Farre R, Lagarda J. Selenium, zinc and copper in plasma of patients with type 1 diabetes mellitus in different metabolic control states. J Trace Elem Med Biol. 1998;12(2):91-95. doi:10.1016/S0946-672X(98)80031-X

25. Bleys J, Navas-Acien A, Guallar E. Serum selenium and diabetes in US adults. Diabetes Care. 2007;30(4):829-834. doi:10.2337/dc061726

26. Ogawa-Wong AN, Berry MJ, Seale LA. Selenium and metabolic disorders: an emphasis on type 2 diabetes risk. Nutrients. 2016;8 (2):80. doi:10.3390/nu8020080

27. Wang XL, Yang TB, Wei J, Lei GH, Zeng C. Association between serum selenium level and type 2 diabetes mellitus: a non-linear dose-response meta-analysis of observational studies. Nutr J. 2016;15(1):48. doi:10.1186/s12937-016-0169-6

28. Rayman MP, Stranges S. Epidemiology of selenium and type 2 diabetes: can we make sense of it? Free Radic Biol Med. 2013;65:1557-1564. doi:10.1016/j.freeradbiomed.2013.04.003

29. Liu X, Zhang Y, Piao J, et al. Reference values of 14 serum trace elements for pregnant chinese women: a cross-sectional study in the China Nutrition and Health Survey 2010-2012. Nutrients. 2017;9 (3):309. doi:10.3390/nu9030309

30. Ghaemi SZ, Forouhari S, Dabbaghmanesh MH, et al. A prospective study of selenium concentration and risk of preeclampsia in pregnant Iranian women: a nested case-control study. Biol Trace Elem Res. 2013;152(2):174-179. doi:10.1007/s12011013-9614-y

31. Maleki A, Fard MK, Zadeh DH, Mamegani MA, Abasaizadeh S, Mazloomzadeh S. The relationship between plasma level of Se and preeclampsia. Hypertens Pregnancy. 2011;30(2):180-187. doi:10.31 09/10641950903322931 


\section{Publish your work in this journal}

The International Journal of General Medicine is an international, peer-reviewed open-access journal that focuses on general and internal medicine, pathogenesis, epidemiology, diagnosis, monitoring and treatment protocols. The journal is characterized by the rapid reporting of reviews, original research and clinical studies

across all disease areas. The manuscript management system is completely online and includes a very quick and fair peer-review system, which is all easy to use. Visit http://www.dovepress.com/ testimonials.php to read real quotes from published authors.

Submit your manuscript here: https://www.dovepress.com/international-journal-of-general-medicine-journal 